\title{
Hardness trends in micron scale indentation
}

\author{
Yueguang Wei ${ }^{\mathrm{a}}$, John W. Hutchinson ${ }^{\mathrm{b}, *}$ \\ ${ }^{a}$ LNM, Institute of Mechanics, Chinese Academy of Sciences, Beijing 100080, China \\ ${ }^{\mathrm{b}}$ Division of Engineering and Applied Sciences, Harvard University, Pierce Hall, \\ Cambridge, MA 02138, USA
}

\begin{abstract}
A continuum theory for elastic-plastic solids that accounts for the size-dependence of strain hardening is employed to analyze trends in the indentation hardness test. Strain gradient plasticity theory incorporates an elevation of flow stress when non-uniform plastic deformations occur at the micron scale. Extensive experimental data exists for size-dependence of indentation hardness in the micron range for conical (pyramidal) indenters, and recent data delineates trends for spherical indenters. Deformation induced by rigid conical and spherical indenters is analyzed in two ways: by exploiting an approximation based on spherically symmetric void expansion and by finite element computations. Trends are presented for hardness as a function of the most important variables in the indentation test, including the size of the indent relative to the material length parameters, the strain hardening exponent, the ratio of initial yield stress to Young's modulus, and the geometry of the indenter. The theory rationalizes seemingly different trends for conical and spherical indenters and accurately simulates hardness data presented recently for iridium, a low yield strain/high hardening material. The dominant role of one of the material length parameters is revealed, and it is suggested that the indentation test may the best means of measuring this parameter.
\end{abstract}

(C) 2003 Elsevier Ltd. All rights reserved.

Keywords: Hardness; Indentation test; Strain hardening; Strain gradient plasticity; Material length parameters

\section{Introduction}

Indentation is used extensively to measure the hardness of metals and polymers. The objective is usually to draw inferences about the mechanical properties (e.g., modulus, yield strength) of the material. Tabor (1951) and Johnson (1970) established relationships between hardness as measured by conical (or pyramidal) and spherical indenters

\footnotetext{
* Corresponding author. Tel.: +1-617-495-2848; fax: +1-617-495-9837.

E-mail address: hutchinson@husm.harvard.edu (J.W. Hutchinson).
} 
and stress-strain behavior in the regime of "large" indents for which conventional plasticity theory is applicable. More recent experiments (Stelmashenko et al., 1993; Ma and Clarke, 1995) have documented a strong size dependence of indentation hardness for micron-size indents that cannot be accounted for by conventional plasticity. Indentation size effect has been one of the motivations underlying the development of a plasticity theory for the micron scale. Conical or pyramidal indents whose widths exceed tens of microns generally produce size-independent hardness values in most metals and can be considered as "large" indents. Smaller indents in the range from sub-micron to about $10 \mu \mathrm{m}$ in single crystals or fine-grained polycrystals often display a significant size effect. The hardness inferred from pyramidal indents on the order of $1 \mu \mathrm{m}$ in width can be two or three times the hardness obtained from an indent that is $10 \mu \mathrm{m}$ across. A clear understanding of the size effect and its connection between material strength is especially important in modern applications involving thin films and multilayers (Freund and Suresh, 2003) since nano- and micro-indentation are frequently the only means of measuring strength.

Recent experiments exploring size dependence associated with indentation by hard spheres (Swadener et al., 2002) have revealed somewhat more complicated behavior than for a pyramidal indenter: at fixed width of indent, hardness increases with decreasing indenter radius, but at a fixed indenter radius, hardness is relatively independent of the indent radius. Experiments on torsion of fine copper wires (Fleck et al., 1994) and bending of thin nickel sheets (Stolken and Evans, 1998) reveal large strength elevations for wire radii and sheet thicknesses in the range from microns to tens of microns. Assuming surface anomalies can be excluded, any explanation of these trends, as well as those for indentation, brings to the fore a material length scale - otherwise the size effect can be excluded by dimensional considerations alone. Strength elevation is believed to be associated with the proliferation of geometrically necessary dislocations induced by strain gradients imposed on the solid (Ashby, 1970; Fleck et al., 1994; Nix and Gao, 1998). Micron scale, non-uniform deformations produce densities of geometrically necessary dislocations that are comparable to, or even exceed, densities of statistically stored dislocations. The smaller is the scale of the (non-uniform) deformation, the larger is the relative density of the geometrically necessary dislocations and the greater is their contribution to hardening.

Micron scale deformations such as those referred to above involve too many dislocations to allow for reliable predictions of behavior based on current formulations of dislocation mechanics. Moreover, deformation behavior in the size range from sub-micron to tens of microns merges smoothly with predictions from conventional "large-scale" plasticity. Thus, there is a compelling motivation for extensions of conventional plasticity that include strain gradient effects. A wide range of micron scale applications has already been investigated within the framework of continuum strain gradient plasticity (Fleck and Hutchinson, 1997; Hutchinson, 2000). One drawback of higher-order formulations of strain gradient plasticity is the inherent difficulty associated with their finite element implementation for numerical analysis. A recent reformulation of phenomenological strain gradient plasticity (Fleck and Hutchinson, 2001) has been proposed which generalizes classical isotropic strain hardening theory and which appears to have the scope to encompass a wide range of deformational behaviors. The new formulation 
gives quantitatively similar predictions for plastic behavior to earlier versions such as those of Fleck and Hutchinson (1997) and Gao et al. (1999), but it improves on the earlier versions by being considerably easier to implement for numerical work.

The new formulation will be employed here to analyze micron scale indentation. Specifically, indentation of an elastic-plastic solid by rigid conical and spherical indenters will be analyzed with emphasis on indentation hardness as dependent on the size of the indent and indenter (in the case of the sphere) relative to the material length parameters, strain hardening, and yield stress to modulus ratio. Approximate theoretical trends will be generated using an approach with makes use of spherically symmetric void expansion in the strain gradient solid, generalizing a method previously demonstrated to be highly effective for large-scale indentation of conventional elastic-plastic solids (Marsh, 1964; Johnson, 1970). In parallel to the approximate results, finite element simulations will also be presented and applied to the recent data of Swadener et al. (2002). The strain gradient plasticity formulation brings in three possible material length parameters, but both approaches reveal that only one of these parameters is important in indentation. A case will be made that indentation is probably the best test for measuring this length parameter.

The present study builds on several earlier theoretical investigations of indentation within the framework of strain gradient plasticity. Nix and Gao (1998) used dislocation mechanics to estimate the density of geometrically necessary dislocations around a conical indenter and the consequent elevation of hardness. While their analysis was qualitative, their study provides a bridge from discrete dislocation mechanics to continuum plasticity providing insights into the phenomenological theory of strain gradient plasticity. The approach of Gao and Nix was extended to spherical indentation by Swadener et al. (2002). In addition to these two studies, the present work complements three recent investigations of indentation hardness based on continuum formulations of strain gradient plasticity that employ finite element analysis to make predications (Begley and Hutchinson, 1998, Huang et al., 2000, Xue et al., 2002).

The outline of the paper is as follows. The new constitutive model for strain gradient plasticity is summarized in Section 2. Details of the indentation model are spelled out in Section 3, followed by a brief discussion of the finite element implementation. The approximate approach based on the solution for the spherical expansion of a void is given in Section 4, while numerical simulations follow in Section 5. Calculations specific to spherical and conical indentation of iridium are given in Section 6, with concluding remarks in Section 7.

\section{Strain gradient plasticity constitutive model}

The constitutive model is a phenomenological generalization of isotropic strain hardening plasticity, commonly referred to as $J_{2}$ theory. The generalization introduces dependence on gradients of plastic strain and on as many as three material length parameters. The deformation theory version of the theory is used rather than the flow theory version because it is easier to implement numerically and because it gives essentially identical predictions to the flow theory when stressing is nearly proportional, 
as is the case for indentation. Comparisons and assessments of the deformation and flow versions are given by Fleck and Hutchinson (2001).

The elastic properties of the material are isotropic with Young's modulus, E, Poisson's ratio, $v$, and tensor of moduli, $C_{i j k l}$. As in conventional small strain theory, the strain is split into elastic and "plastic" parts according to $\varepsilon_{i j}=\varepsilon_{i j}^{\mathrm{e}}+\varepsilon_{i j}^{\mathrm{P}}$ with $\varepsilon_{j j}^{\mathrm{P}}=0$. The conventional stresses, $\sigma_{i j}=\sigma_{j i}$, satisfy $\sigma_{i j}=C_{i j k l} \varepsilon_{k l}^{\mathrm{e}}$. Let $m_{i j}=(3 / 2) s_{i j} / \sigma_{\mathrm{e}}$ with $s_{i j}$ as the deviator of $\sigma_{i j}$ and $\sigma_{\mathrm{e}}=\sqrt{3 s_{i j} s_{i j} / 2}$ as the conventional effective stress. Take $\varepsilon_{\mathrm{P}}$ to be the non-negative amplitude of the "plastic" strain, $\varepsilon_{\mathrm{P}}=\sqrt{2 \varepsilon_{i j}^{\mathrm{P}} \varepsilon_{i j}^{\mathrm{P}} / 3}$. The "normality" condition $\varepsilon_{i j}^{\mathrm{P}}=\varepsilon_{\mathrm{P}} m_{i j}$ is enforced. Higher-order stress quantities, $\tau_{i}$, which are work conjugate to $\varepsilon_{\mathrm{P}, i}$, are present in the theory and are given below. As in conventional $J_{2}$ theory, the uniaxial stress-strain curve of the material is an input to the constitutive model, and here $\sigma\left(\varepsilon_{\mathrm{P}}\right)$ is used to denote the stress-plastic strain relation in uniaxial tension.

The gradients of the plastic strains are $\rho_{i j k}=\rho_{j i k}=\varepsilon_{i j, k}^{\mathrm{P}}$, and a generalized effective plastic strain is defined as

$$
E_{\mathrm{P}}^{2}=\varepsilon_{\mathrm{P}}^{2}+\ell_{1}^{2} I_{1}+4 \ell_{2}^{2} I_{2}+(8 / 3) \ell_{3}^{2} I_{3} .
$$

The three quadratic invariants, $I_{n}$, of $\rho_{i j k}$ in Eq. (1) are defined as follows (Fleck and Hutchinson, 2001). With $e_{i j k}$ as the permutation tensor, let

$$
\rho_{i j k}^{S}=\frac{1}{3}\left(\rho_{i j k}+\rho_{j k i}+\rho_{k i j}\right), \quad \chi_{i j}=e_{i q r} \rho_{j r q} .
$$

Then,

$$
\begin{aligned}
& I_{1}=\rho_{i j k}^{S} \rho_{i j k}^{S}-\frac{4}{15} \rho_{k i i} \rho_{k j j}, \\
& I_{2}=\frac{1}{3}\left(\chi_{i j} \chi_{i j}+\chi_{i j} \chi_{j i}\right), \\
& I_{3}=\frac{3}{5}\left(\chi_{i j} \chi_{i j}-\chi_{i j} \chi_{j i}\right) .
\end{aligned}
$$

The generalized effective plastic strain is intended as a phenomenological measure of the total dislocation density: statistically stored dislocations are due to $\varepsilon_{\mathrm{P}}$ and geometrically necessary dislocations are induced by the plastic strain gradients. Expression (1) is the most general isotropic measure that is homogeneous of degree two in the plastic strains and their first gradients. It is positive definite, and the three length parameters, $\ell_{i}$, are required for dimensional consistency. These length parameters set the scales at which the gradients become important. Of the three parameters, $\ell_{1}$ plays the dominant role in indentation. However, for application to a wide range of micron scale phenomena, it is essential that at least two of the length parameters be retained in the theory, as argued in Fleck and Hutchinson (2001).

From $\varepsilon_{i j}^{\mathrm{P}}=\varepsilon_{\mathrm{P}} m_{i j}$, it follows that $\rho_{i j k} \equiv \varepsilon_{i j, k}^{\mathrm{P}}=\varepsilon_{\mathrm{P}, k} m_{i j}+\varepsilon_{\mathrm{P}} m_{i j, k}$. Upon substitution into Eqs. (1)-(3), one obtains an expression for the generalized plastic strain in terms of $\varepsilon_{\mathrm{P}}$ and $\varepsilon_{\mathrm{P}, i}$ as

$$
E_{\mathrm{P}}^{2}=\varepsilon_{\mathrm{P}}^{2}+A_{i j} \varepsilon_{\mathrm{P}, i} \varepsilon_{\mathrm{P}, j}+B_{i} \varepsilon_{\mathrm{P}, i} \varepsilon_{\mathrm{P}}+C \varepsilon_{\mathrm{P}}^{2} .
$$

The factors $A_{i j}=A_{j i}, B_{i}$ and $C$ are given by Fleck and Hutchinson (2001); they depend on the material length parameters and on $m_{i j}$ and its gradients. 
In analogue to classical deformation theory, a potential energy functional specifies the generalized strain gradient theory. The following potential reduces to the classical functional when gradient effects can be ignored, or, equivalently, if the length parameters are set to zero. This ensures that the limit of the generalized theory coincides with the classical theory in the limit when the scale of the deformation is large compared to the material length parameters. The potential is defined as

$$
\begin{aligned}
\Phi\left(u_{i}, \varepsilon_{\mathrm{P}}\right)= & \int_{V}\left\{\frac{1}{2} C_{i j k l}\left(\varepsilon_{i j}-\varepsilon_{\mathrm{P}} m_{i j}\right)\left(\varepsilon_{k l}-\varepsilon_{\mathrm{P}} m_{k l}\right)+\int_{0}^{E_{\mathrm{P}}} \sigma\left(\tilde{\varepsilon}_{\mathrm{P}}\right) d \tilde{\varepsilon}_{\mathrm{P}}\right\} \mathrm{d} V \\
& -\int_{S_{T}}\left(T_{i}^{0} u_{i}+t^{0} \varepsilon_{\mathrm{P}}\right) \mathrm{d} S .
\end{aligned}
$$

Here, $V$ denotes the volume of the body, $S$ its surface with $n_{i}$ as its outward unit normal. On the portion of the surface, $S_{T}$, traction is prescribed with $T_{i}^{0}$ as the conventional traction and $t^{0}$ as a higher-order traction defined below. As defined earlier, $\sigma\left(\varepsilon_{\mathrm{P}}\right)$ denotes data from the uniaxial tensile stress-strain curve of the material. The first contribution to the volume integral in Eq. (5) is the elastic energy density while the second is the plastic work density, now evaluated at $E_{\mathrm{P}}$ rather than $\varepsilon_{\mathrm{P}}$. It is through this term that the dependence on the plastic strain gradients enters. The generalized plastic work density provides the clearest and most elementary insight into the role played by $E_{\mathrm{P}}$ in the theory. Simply stated, the theory postulates that the plastic work expended in bring a material element subject to both plastic strains and plastic strain gradients to a generalized strain $E_{\mathrm{P}}$ is the same as occurs in deforming that element to the plastic strain $\varepsilon_{\mathrm{P}}=E_{\mathrm{P}}$ in uniaxial tension.

Provided the function $\sigma\left(\varepsilon_{\mathrm{P}}\right)$ is monotonically increasing, a unique solution to any boundary value problem can be obtained by minimizing $\Phi$ with respect to all admissible $u_{i}$ and $\varepsilon_{\mathrm{P}}$. The field equations associated with stationarity of $\Phi$ are

$$
\begin{aligned}
& \sigma_{i j, j}=0, \\
& Q \equiv \sigma_{\mathrm{e}}+\tau_{i, i}=\frac{\sigma\left(E_{\mathrm{P}}\right)}{E_{\mathrm{P}}}\left(\varepsilon_{\mathrm{P}}+\frac{1}{2} B_{i} \varepsilon_{\mathrm{P}, i}+C \varepsilon_{\mathrm{P}}\right), \\
& \tau_{i}=\frac{\sigma\left(E_{\mathrm{P}}\right)}{E_{\mathrm{P}}}\left(A_{i j} \varepsilon_{\mathrm{P}, j}+\frac{1}{2} B_{i} \varepsilon_{\mathrm{P}}\right) .
\end{aligned}
$$

The boundary relations between the stress and traction quantities on $S$ are

$$
T_{i}=\sigma_{i j} n_{j} \quad \text { and } \quad t=\tau_{i} n_{i} .
$$

The virtual work statement for this class of theories is

$$
\int_{V}\left\{\sigma_{i j} \delta \varepsilon_{i j}^{\mathrm{e}}+Q \delta \varepsilon_{\mathrm{P}}+\tau_{i} \delta \varepsilon_{\mathrm{P}, i}\right\} \mathrm{d} V=\int_{S}\left(T_{i} \delta u_{i}+t \delta \varepsilon_{\mathrm{P}}\right) \mathrm{d} S .
$$

Here, $\sigma_{i j} \delta \varepsilon_{i j}^{\mathrm{e}}$ is the elastic work increment, $Q$ in Eq. (7) is work conjugate to the plastic strain $\varepsilon_{\mathrm{P}}$, and the vector quantity $\tau_{i}$ is the higher-order stress working through the plastic strain gradient $\varepsilon_{\mathrm{P}, i}$. The contributions in Eqs. (5) and (10) from $t$ and $\tau_{i}$ are absent if $E_{\mathrm{P}}=0$ or if the material length parameters are zero. 


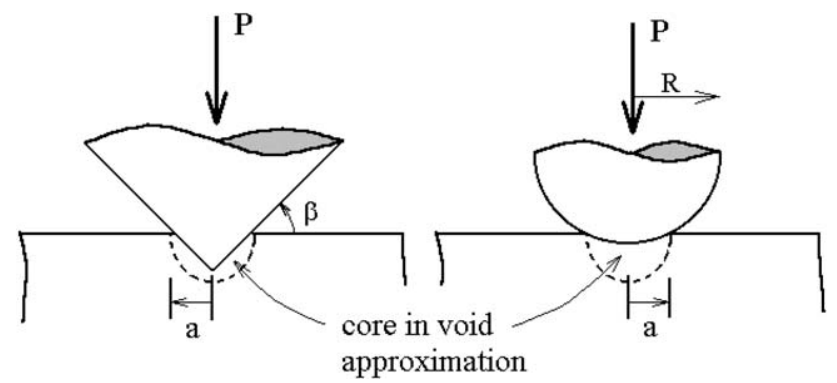

Fig. 1. Geometry of rigid conical and spherical indentation. The hemispherical core region directly under the contact region is used in carrying out the indentation analysis using the void expansion approximation.

The formulation is unusual in the sense that the free variables employed are $u_{i}$ and $\varepsilon_{\mathrm{P}}$. It is this feature that makes the formulation easier to implement in numerical work than earlier formulations. Although the variational principle based on $\Phi$ is not standard because of the explicit role of $\varepsilon_{\mathrm{P}}$ as a variable, the formulation does reduce to the classical $J_{2}$ theory when the material length parameters vanish.

\section{The indentation problems}

The indenter is either a rigid cone with angle $\beta$ or a rigid sphere of radius $R$, as depicted in Fig. 1. The indenter is loaded with a normal force $P$, and the hardness is defined as $H=P /\left(\pi a^{2}\right)$, where $a$ is the radius of contact of the indenter. The substrate is semi-infinite and is characterized by the constitutive model detailed in Section 2. The problem is axisymmetric. The tensile stress-strain curve of the substrate is taken to be the Ramberg-Osgood relation

$$
\varepsilon=\frac{\sigma}{E}+\alpha \frac{\sigma_{\mathrm{Y}}}{E}\left(\frac{\sigma}{\sigma_{\mathrm{Y}}}\right)^{n}
$$

with $\sigma_{\mathrm{Y}}$ as the nominal yield stress. The function appearing in the potential $\Phi$ is, therefore, $\sigma\left(\varepsilon_{\mathrm{P}}\right)=\sigma_{\mathrm{Y}}\left(\varepsilon_{\mathrm{P}} /\left(\alpha \varepsilon_{\mathrm{Y}}\right)\right)^{N}$ with $\varepsilon_{\mathrm{Y}}=\sigma_{\mathrm{Y}} / E$ and $N=1 / n$ as the strain hardening exponent. With specification of the three length parameters, $\ell_{i}$, the solid is fully prescribed. All numerical results presented in this paper based on Eq. (11) are computed with $\alpha=\frac{3}{7}$.

\subsection{Finite element method}

The semi-infinite half-space is replaced by a finite hemispherical substrate whose radius, $R_{0}$, is very large compared to the radius of the indent, $a$, and the surrounding plastic zone. Tractions are applied on the outer hemispherical surface corresponding to those for a concentrated load, $P$, applied normally to an infinite elastic half-space at radial distance $R_{0}$ from the point of application (Timoshenko and Goodier, 1970). These tractions equilibrate the applied load and specify the correct asymptotic stresses 
for the semi-infinite problem. Frictionless contact is assumed at the common surface between the indenter and the substrate. Moreover, the plastic strain $\varepsilon^{\mathrm{P}}$ is assumed to be unconstrained at the contact surface such that the higher-order boundary condition enforced by the variational solution is $t=0$. A few simulations were carried out with ${ }_{\varepsilon}^{\mathrm{P}}=0$ at the contact surface, but the hardness predictions were only slightly changed from the results with unconstrained plastic flow at the contact surface. A finite element code for axisymmetric deformations has been developed for the strain gradient plasticity formulation of Section 2. At each node, there are three nodal variables, the two displacement components and the plastic strain amplitude $\varepsilon^{\mathrm{P}}$. These three variables are expressed within the elements by shape functions. Stress gradients appear in the expression for the effective strain $E_{\mathrm{p}}$ in Eq. (4) through the coefficients $B_{i}$ and $C$; these are calculated directly through the constitutive equation with the aid of the shape functions. Selected details related to the constitutive relation are outlined in Appendix A.

\subsection{Spherically symmetric void expansion}

The spherically symmetric solution (Fleck and Hutchinson, 2001) for growth of a spherical void containing a hydrostatic pressure, $p$, in an infinite solid was used a benchmark to demonstrate the accuracy of the axisymmetric finite element program formulation. For conventional plasticity, spherically symmetric void expansion has been used to develop approximate indentation solutions (Johnson, 1970). The corresponding solution for strain gradient plasticity will be used for this same purpose in the next section, and in this respect it is a particularly sensible choice for benchmarking the present program. The solid is also specified by the deformation theory version of the strain gradient plasticity theory in Section 2 for a material with the Ramberg-Osgood tensile stress-strain curve (11) with $\alpha=\frac{3}{7}$. A relatively simple exact formulation for generating a numerical solution to the problem was given in Fleck and Hutchinson (2001) for the limiting case of an elastically incompressible solid, $v=\frac{1}{2}$. The solution is obtained within the framework of small strain theory. The second of the three material lengths, $\ell_{2}$, does not enter the solution for this problem. The initial radius of the void is $a$, the initial volume, $V=4 \pi a^{3} / 3$, and the volume expansion is denoted by $\Delta V$. Plastic flow is unconstrained at the void surface such that the higher-order boundary condition is $t=0$.

A selected set of numerical results generated using the scheme detailed in Fleck and Hutchinson (2001) is presented in Fig. 2 as $p / \sigma_{\mathrm{Y}}$ against $\Delta V /\left(\varepsilon_{\mathrm{Y}} V\right)$ for $N=0.2$ and combinations of $\ell_{1}$ and $\ell_{3}$. Presented in this form, the results are independent of $\sigma_{\mathrm{Y}} / E$. One also notes that there is only a very weak dependence of the void expansion solution on $\ell_{3}$-an order of magnitude change of this parameter has little influence. Included in Fig. 2 are results computed using the axisymmetric finite element program developed for the indentation study. Elastic compressibility is required in this program, and results have been computed for both $v=0.3$ and 0.48 , with the latter falling quite close to the results for the elastically incompressible material. Spherical symmetry was not invoked in the finite element calculation but symmetry with respect to the equatorial plane was used. A total of 720 quadrilateral elements were used with 9 


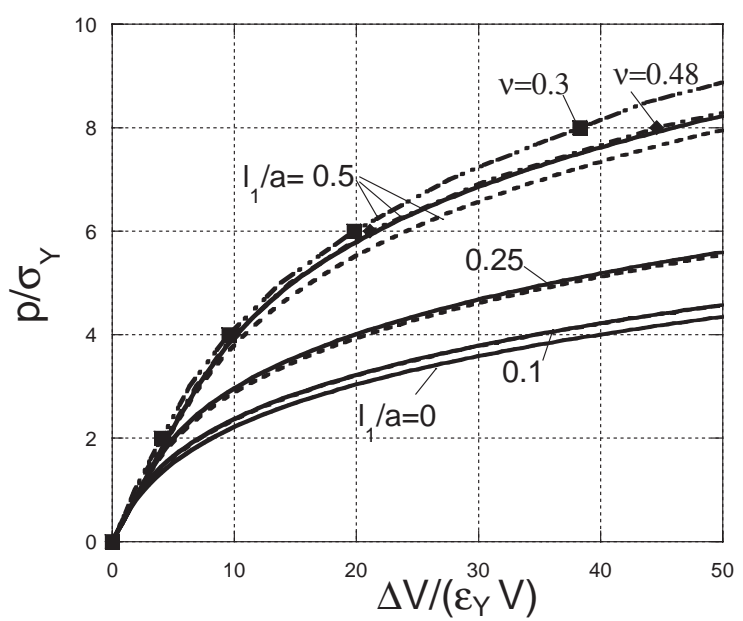

Fig. 2. Expansion of a spherical void in an infinite solid subject to internal pressure. The curves without markers are for a solid that is elastically incompressible and characterized by the strain gradient plasticity with material length parameters $\ell_{1}=\ell_{3}$ (solid curves) and $\ell_{3}=\ell_{1} / 10$ (dashed curves). There is no dependence on $\ell_{2}$. Uniaxial tensile behavior is given by the Ramberg-Osgood relation with $N=0.2$ and $\alpha=\frac{3}{7}$. The two curves with markers have been computed using the axisymmetric finite element code developed for the present study for elastically compressible solids with $\ell_{1}=\ell_{3}=\ell_{2}$.

nodes/element and 3 variables/node. Gauss integration was employed with $2 \times 2$ points per element.

\section{Hardness trends based on the void expansion approximation}

Johnson (1970), following the earlier ideas of Marsh (1964), demonstrated the efficacy of predictions of indentation hardness derived from the solution for spherical void expansion. They approximated the field around "large" indents using the solution for spherically symmetric void expansion obtained on the basis of conventional plasticity. Here, this same approach will be implemented for strain gradient plasticity and used to illustrate hardness trends with particular emphasis on the size dependence.

The solution for the spherically symmetric expansion of a spherical void in the elastically incompressible solid discussed in the previous section can be written in terms of the dimensionless variables and parameters as

$$
\frac{p}{\sigma_{\mathrm{Y}}}=f\left[\frac{\Delta V}{\varepsilon_{\mathrm{Y}} V}, N, \frac{a}{\ell_{1}}\right] \text {. }
$$

There is no dependence on $\ell_{2}$, and the weak dependence on $\ell_{3}$ is not noted. All results presented below based on the void approximation have been determined with $\ell_{3}=\ell_{1}$.

The indentation approximation is motivated using Fig. 1 and the rational employed by Johnson (1970). Directly under the indenter contact area in a hemispherical core of radius $a$, the solid is assumed to be in a state of uniform hydrostatic pressure such 
that $p=H$, since the hardness, $H$, is by definition the average normal pressure under the indenter. The field outside the core is approximated by the spherically symmetric solution for void expansion, with the core as the spherical void of radius $a$ containing pressure $p$. The volume of the indent is identified with one-half the expansion of the void, $\Delta V / 2$. For the conical indenter, $\Delta V=(2 \pi / 3) \tan \beta a^{3}$, and thus, by Eq. (12), the hardness relation is

$$
\frac{H}{\sigma_{\mathrm{Y}}}=f\left[\frac{\tan \beta}{2 \varepsilon_{\mathrm{Y}}}, N, \frac{a}{\ell_{1}}\right] \quad \text { (conical indenter). }
$$

For relatively shallow indents with the spherical indenter, $\Delta V=(\pi / 2) a^{4} / R$, and, by Eq. (12),

$$
\frac{H}{\sigma_{\mathrm{Y}}}=f\left[\frac{3 a}{8 \varepsilon_{\mathrm{Y}} R}, N, \frac{a}{\ell_{1}}\right] \quad \text { (spherical indenter). }
$$

The Marsh-Johnson approximations correspond to $a / \ell_{1} \rightarrow \infty$. Approximations (13) and (14) draw no distinction between the size of indents in the loaded and unloaded state, nor does it account for the influence of pile-up or depression around the perimeter of the indent or indenter elasticity. These are important secondary effects that are generally taken into account in indentation hardness testing protocols. Nevertheless, it will be shown that Eqs. (13) and (14) capture the first-order hardness relations quite effectively.

For the purpose of generating numerical results based on Eqs. (13) and (14), the following approximate power law interpolation of the spherical void solution (12) has been developed, guided by the form of the Ramberg-Osgood stress-strain curve (1):

$$
\frac{\Delta V}{\varepsilon_{\mathrm{Y}} V}=\frac{9}{4} \frac{p}{\sigma_{\mathrm{Y}}}+\alpha\left(\frac{p}{b\left(N, a / \ell_{1}\right) \sigma_{\mathrm{Y}}}\right)^{n} .
$$

This representation is exact in the linear range and it has the correct power law functional form in the limit that $p / \sigma_{\mathrm{Y}}$ becomes large. The coefficient $b$ has been chosen such that $p / \sigma_{\mathrm{Y}}$ from Eq. (15) coincides at $\Delta V /\left(\varepsilon_{\mathrm{Y}} V\right)=50$ with the exact solution, computed in the manner described in the previous section. Curves of $b\left(N, a / \ell_{1}\right)$ are presented in Fig. 3, again with $\alpha=\frac{3}{7}$. The function $f$ in (12) is obtained by numerically solving Eq. (15) for $p / \sigma_{\mathrm{Y}}$ at any given $\Delta V /\left(\varepsilon_{\mathrm{Y}} V\right)$. The curves in Fig. 4 are obtained from Eq. (15) and can be compared to those presented earlier in Fig. 2. Apart from some discrepancy in the relatively narrow transition between linear and nonlinear regions, interpolation (15) provides an approximation to the spherical expansion solution that is sufficiently accurate for present purposes.

\subsection{Hardness trends for conical indenters}

The implication of Eq. (13), that $H / \sigma_{\mathrm{Y}}$ depends on indenter angle and yield strain in combination, $\tan \beta / \varepsilon_{\mathrm{Y}}$, extends to the micron scale the outcome of the earlier studies (e.g. Johnson, 1970). The two plots of Fig. 5 based on approximation (13) summarize the parametric dependence of the normalized hardness. As noted in earlier studies (e.g. Begley and Hutchinson, 1998; Nix and Gao, 1998; Elmustafa and Stone, 2003), a strong size dependence is predicted for conical indentation with the hardness, $H$, diminishing to the limit for large indents as the radius $a$ of the indent increases. For the present 


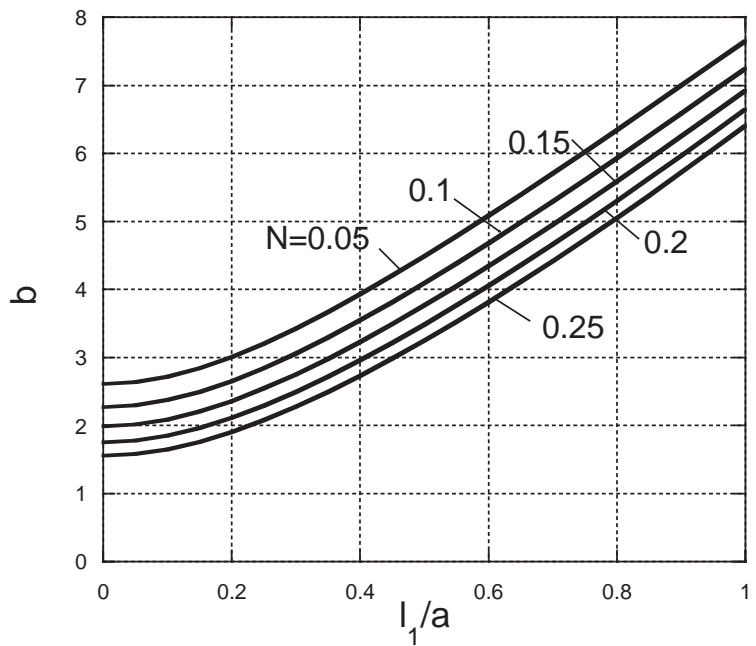

Fig. 3. Coefficient $b\left(N, \ell_{1} / a\right)$ in void expansion approximation (15). Computed with $\ell_{3}=\ell_{1}$.

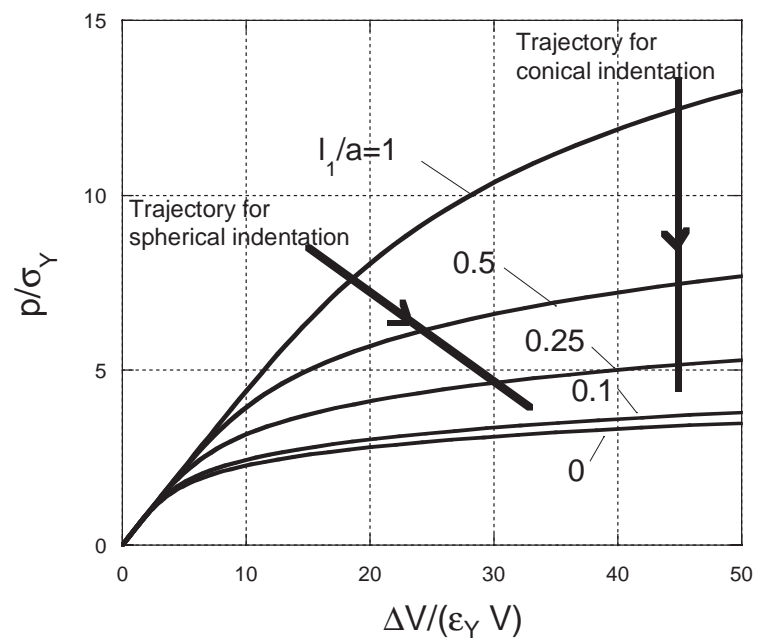

Fig. 4. Expansion of a spherical void from the power law approximation (15) with $N=0.2$ and $\alpha=\frac{3}{7}$. The approximate results can be compared with the corresponding curves from the exact solution in Fig. 2. Trajectories under increasing indent size are traced typical of those for conical and spherical indentation.

theory, the size dependence becomes evident for $a / \ell_{1}$ less than about 10 , and elevations in hardness on the order of a factor of roughly 2 occur when $a / \ell_{1}=2$. The result for a large indent in an elastic-perfectly plastic solid is $H / \sigma_{\mathrm{Y}}=\frac{2}{3}\left(1+\ln \left(\tan \beta / 3 \varepsilon_{\mathrm{Y}}\right)\right)$ (Johnson, 1970), in agreement with the limit for large indents for the solid with low strain hardening in Fig. 5. 


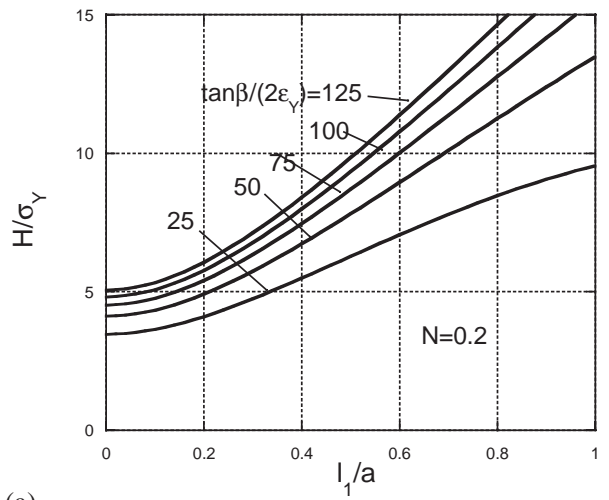

(a)

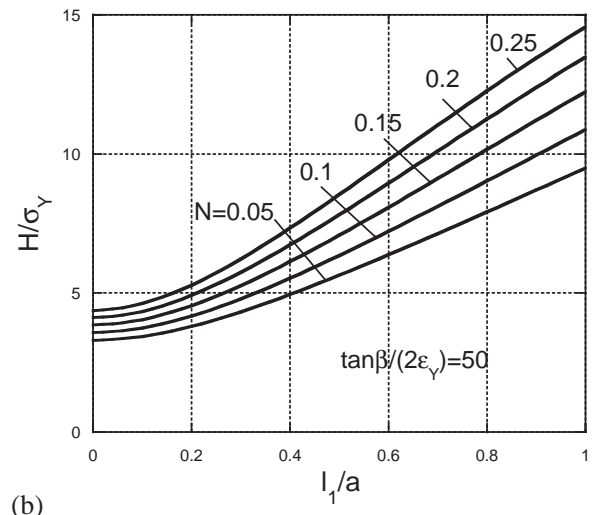

(b)

Fig. 5. Hardness trends for conical indentation from the void expansion approximation.

\subsection{Hardness trends for spherical indenters}

The convenient separation of geometry and yield strain via a single parameter in conical indentation does not hold for spherical indentation, as can be seen from Eq. (14). Trends for spherical indentation are inherently more complicated. For present purposes, the first set of trends will be presented as a function of $a / R$, in the manner that recent experiment data has been presented by Swadener et al. (2002). Rewrite Eq. (14) in a form that emphasizes the dependence on $a / R$ as

$$
\frac{H}{\sigma_{\mathrm{Y}}}=f\left[\frac{3 a}{8 \varepsilon_{\mathrm{Y}} R}, N, \frac{a}{R} \frac{R}{\ell_{1}}\right] \quad \text { (spherical indenter). }
$$

Plots of normalized hardness as a function of $a / R$ are presented in Fig. 6. From these plots, as well as Eq. (16), it is clear that $R / \ell_{1}, N$ and $\varepsilon_{Y}$ have independent effects. The uppermost plot in Fig. 6 reveals that the dominant size effect in spherical indentation is associated with variations in indenter size, $R$, rather than, $a / R$, as emphasized by Swadener et al. (2002). For $a / R$ sufficiently large such that plastic deformation under the indenter dominates elastic deformation (i.e. for $\left.a /\left(\varepsilon_{Y} R\right)>25\right)$, there is a relatively weak dependence on $a / R$. For smaller $a / R$, the hardness trend with $a / R$ at fixed $R / \ell_{1}$ would appear to be opposite that for conical indentation. However, the regime in which $H / \sigma_{\mathrm{Y}}$ decreases with decreasing $a / R$ corresponds to the region of transition in $f$ from elastic to plastic void growth in Fig. 2 . This is the regime in which plasticity does not dominant behavior under the indenter. The void approximation is expected to suffer its largest errors in this regime. Nevertheless, the trend in hardness with $a / R$ in Fig. 6 is similar to that seen in the data taken by Swadener et al. (2002) and from their model.

The dependence of $H / \sigma_{\mathrm{Y}}$ on $N$ and $\varepsilon_{\mathrm{Y}}=\sigma_{\mathrm{Y}} / E$ is presented in two lower plots in Fig. 6. Both low strain hardening and low yield strain can lead to falling hardness with increasing indentation depth, but the effect is weak compared to that for conical indentation. For all curves in Fig. 6 , the range of $a / \ell_{1}=(a / R)\left(R / \ell_{1}\right)$ has been limited to $a / \ell_{1} \geqslant 1$; termination of the curves on their left end corresponds to $a / \ell_{1}=1$. 

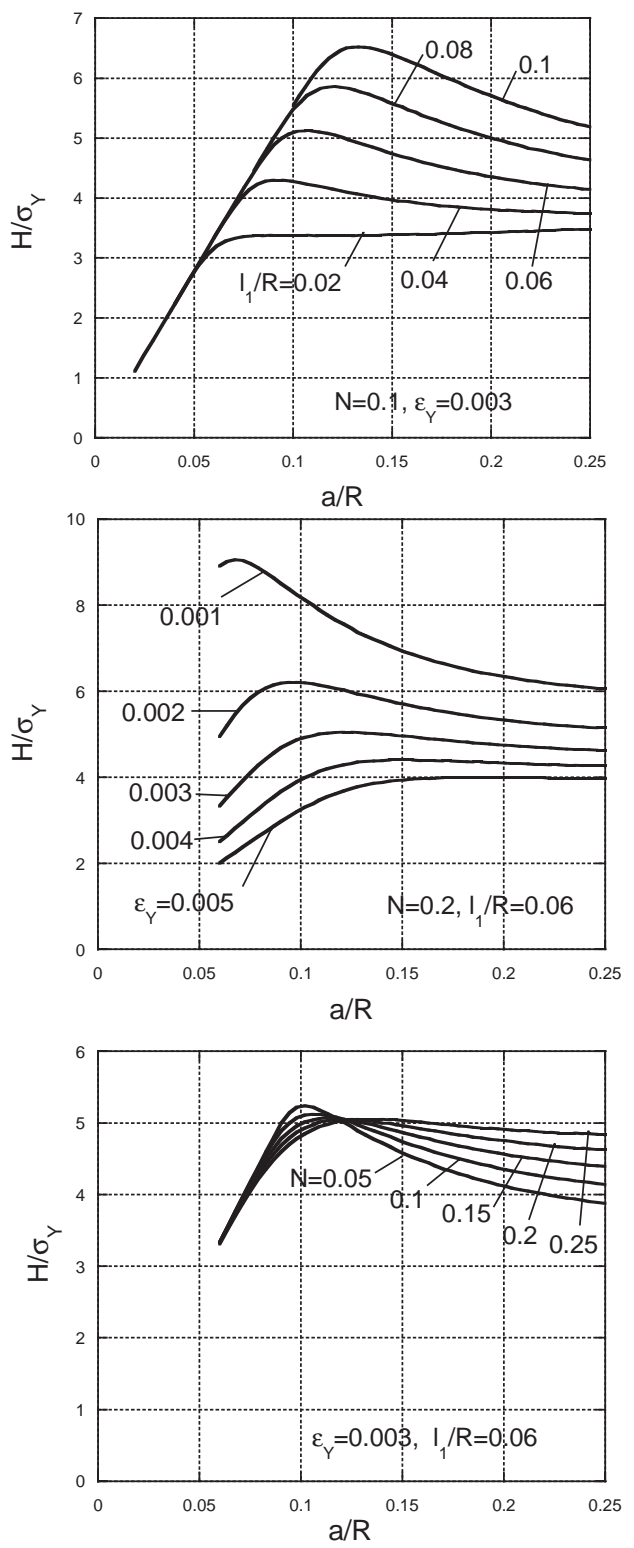

Fig. 6. Hardness trends for spherical indentation from the void expansion approximation.

The similarity in the size dependence of spherical indenters with that for conical indenters is brought out by the curves of $H / \sigma_{\mathrm{Y}}$ against $\ell_{1} / R$ for fixed $a / R$ in Fig. 7. The limit for large size-independent indents is attained for $\ell_{1} / R<0.01$. Thus, if $\ell_{1} \approx 1 \mu \mathrm{m}$, significant size dependence is expected for spherical indenters with radii 


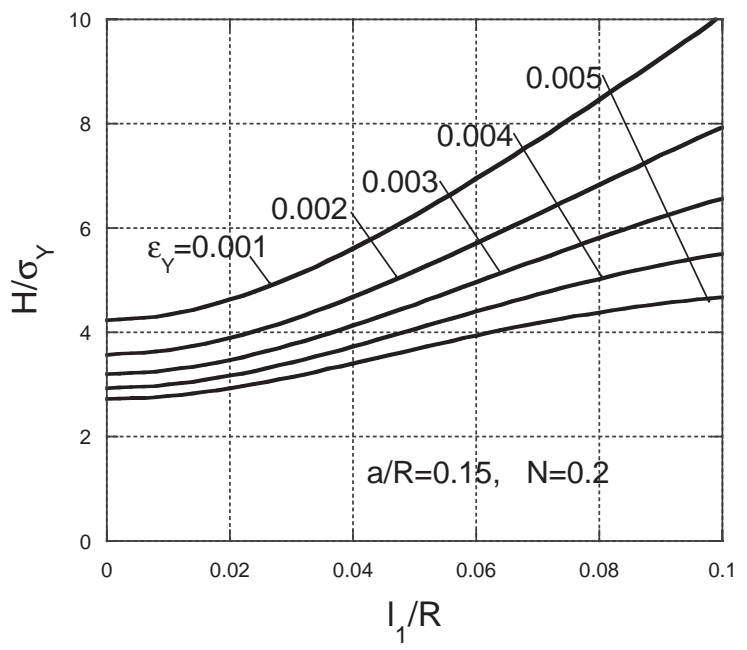

Fig. 7. Hardness trends for spherical indentation from the void expansion approximation. Dependence on size of the spherical indenter at fixed $a / R$.

less than about $100 \mu \mathrm{m}$. This is the case for the data presented for annealed oxygen-free copper by Swadener et al. (2002). Their data for iridium suggests that the transition to size-independent behavior is $R \approx 300 \mu \mathrm{m}$, implying $\ell \approx 3 \mu \mathrm{m}$ for this material, as will be seen to be the case in Section 6 .

\subsection{Conical versus spherical indentation}

Johnson (1970), elaborating on Tabor (1951) proposals, notes that conventional hardness measured for strain hardening materials can be associated with the uniaxial flow stress at a representative strain, $\sigma\left(\varepsilon_{\mathrm{r}}\right)$, according to $H \cong 3 \sigma\left(\varepsilon_{\mathrm{r}}\right)$. The representative strain under a conical indenter is $\varepsilon_{\mathrm{r}} \cong 0.2 \tan \beta$, independent of indent size, while the representative strain under a spherical indenter depends on indent size according to $\varepsilon_{\mathrm{r}} \cong 0.2 a / R$. In other words, data taken in the form of load versus indentation radius, $a$, provides different information for the two shapes of indenters. Distinction between behaviors for the two shapes persists into the micron range. This is illustrated in Fig. 4, where typical trajectories associated with increasing indent size, $a$, are mapped onto the void expansion solution. For the conical indent, $\Delta V /\left(\varepsilon_{\mathrm{Y}} V\right)=\tan \beta /\left(2 \varepsilon_{\mathrm{Y}}\right)$ is constant, while for the spherical indent, $\Delta V /\left(\varepsilon_{\mathrm{Y}} V\right)=3 a /\left(8 \varepsilon_{\mathrm{Y}} R\right)$ increases. In each case, the trajectory shifts across curves for larger and larger voids relative to the material length, $\ell_{1}$, as $a$ increases. For the conical indenter, the effective strain under the indenter is constant and the drop in hardness is due to the increase in indent size. Under the spherical indenter, the hardness drop due to increasing indent size is counteracted by the hardness rise due to increasing strain, $\varepsilon_{\mathrm{r}} \cong 0.2 a / R$.

According to the void expansion approximations, Eqs. (13) and (14), the hardness measured for a particular material by the two indenters should be identical if the radii 
of the indents, $a$, are the same and if $3 a /(4 R)=\tan \beta$, whether in the micron range or in the "large" size range. Increasing $a$ at fixed radius, $R$, for the spherical indenter is equivalent to testing with a sequence of conical indenters of increasing angle $\beta$. Alternatively, increasing $a$ at fixed $\beta$ for the conical indenter is equivalent to testing with a sequence of spherical indenters with larger and larger radii, $R$. Swadener et al. (2002) employed a sequence of spherical indenters of varying $R$ to bring out the strong size dependence of several materials, as will be discussed in Section 6 .

\section{Hardness trends based finite element computations}

A selected number of the trends discussed based on the void expansion approximation were computed using the axisymmetric finite element program developed for the full field equations in Section 2. The dominant importance in indentation of the first material length parameter, $\ell_{1}$, over $\ell_{2}$ and $\ell_{3}$ was verified using the finite element program. For all the calculations reported in this section, the role of $\ell_{1}$ is emphasized, and the calculations have been carried out with $\ell_{3}=\ell_{2}=\ell_{1}$. The tensile stress-strain curve characterizing the solid is the same as that used for the void expansion approximation, i.e. Eq. (11) with $\alpha=\frac{3}{7}$. Poisson's ratio is taken to be $v=0.3$.

The computed hardness trends for the rigid conical indenter are shown in the two parts of Fig. 8. In almost every respect, the trends from the finite element calculations are in reasonably close quantitative agreement with those presented earlier based on the spherical void approximation. First, it can be noted that the implication from the void approximation that the parameter, $\tan \beta / \varepsilon_{Y}$, collapses the dependence on cone angle and initial yield strain is indeed borne out to a very good approximation. Secondly, it can be noted that the dependence on the strain hardening exponent, $N$, in Fig. $8 \mathrm{~b}$ is completely in accord with the corresponding trend based on the void approximation. The main difference between the trends in Fig. 8 and those based on the void approximation in Figs. 4 and 5 is approach to the classical limit for large indents as $\ell_{1} / a \rightarrow 0$. The numerical results in Fig. 8 from the finite element calculations indicate that the hardness approaches the large indent limit nearly linearly with $\ell_{1} / a$, while the void approximation suggests that this limit is approached more slowly, roughly in proportion to $\left(\ell_{1} / a\right)^{2}$. The large indent limits from the two approaches are within $10 \%$ of one another. Over the entire range of $\ell_{1} / a$, the difference between the void approximation and the finite element results is generally less than $10 \%$, with the largest discrepancy occurring at $\ell_{1} / a \approx 0.1$ due to the different approaches to the large indent limit.

The finite element results for the rigid spherical indenter are presented in Fig. 9. The dependence of the normalized hardness, $H / \sigma_{\mathrm{Y}}$, on $a / R$ at fixed values of $\ell_{1} / R$ can be compared directly with the corresponding curves based on the void approximation in upper plot of Fig. 6. In this case, the general trends are again similar, but the discrepancy between the finite element predictions and the void approximation are greater. The finite element results indicate that the hardness has little dependence on $a / R$ at fixed $R / \ell_{1}$ once $a / R$ is larger than about 0.05 , in agreement of the observations and modeling of Swadener et al. (2002). Larger variation with $a / R$ is predicted by the void approximation. It is not surprising that the void approximation looses accuracy when $a / R<0.05$ because this is the range in which plasticity is not dominant under 

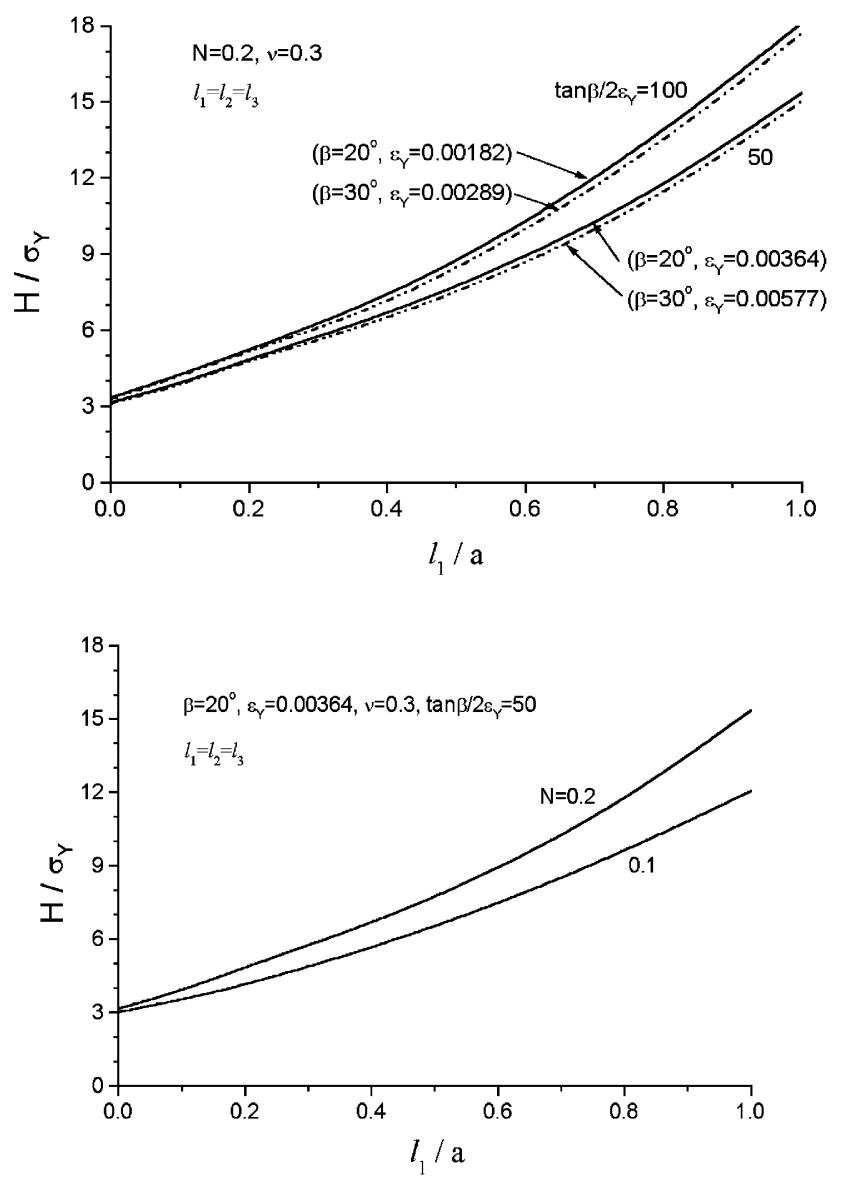

Fig. 8. Hardness trends for conical indentation computed using the finite element program. Top: Variation of cone and angle and initial yield strain showing the significance of the parameter $\tan \beta / \varepsilon_{\mathrm{Y}}$. Bottom: Variation of strain hardening exponent.

the indenter and justification for the void approximation is lost. The strong size effect under spherical indentation is seen in the lower plot in Fig. 9, where hardness is plotted against $\ell_{1} / R$ with $a / R=0.15$ and three values of the initial yield strain. The trends are similar to those from the void approximation in Fig. 7. However, the void approximation exaggerates the spread in the dependence on the initial yield strain, overestimating the hardness at smaller $\varepsilon_{Y}$ and underestimating it a larger $\varepsilon_{Y}$.

\section{Simulations of the data for spherical and pyramidal indentation of iridium}

Swadener et al. (2002) obtain hardness data on fine-grained polycrystalline iridium using both spherical and pyramidal Berkovich indentation. They employed five spherical 

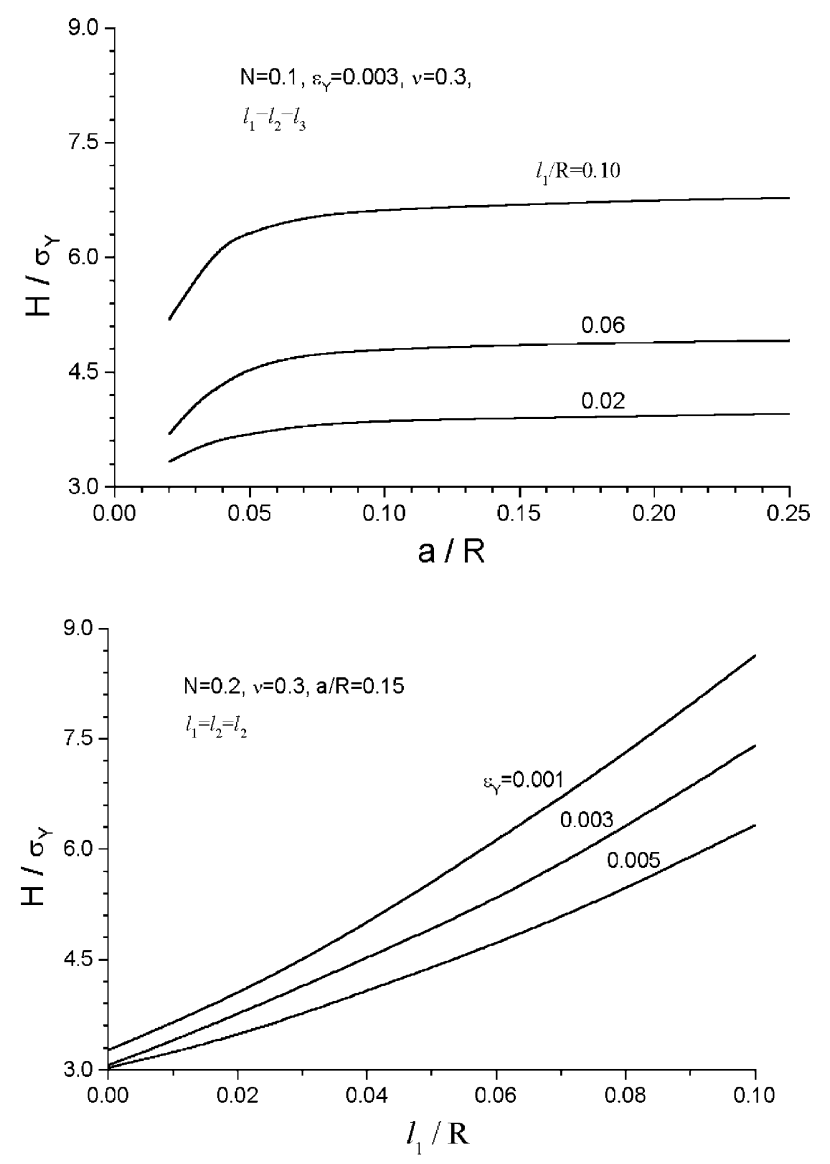

Fig. 9. Hardness trends for spherical indentation computed using the finite element program. Top: Hardness versus $a / R$ at fixed $\ell_{1} / R$. Bottom: Hardness versus $\ell_{1} / R$ at fixed $a / R$.

indenters ranging in radius from 14 to $1600 \mu \mathrm{m}$. Iridium has an exceptionally low initial yield strain (0.00021) and very high strain hardening. Swadener et al., found that for stresses above the yield stress, $\sigma_{\mathrm{Y}}=109 \mathrm{MPa}$, and strains less than about $5 \%$, the tensile stress-strain curve is well approximated by

$$
\sigma=\sigma_{\mathrm{Y}}+k \varepsilon^{N},
$$

where $k=2739 \mathrm{MPa}$ and $N=0.638$. Stress-strain data at larger strains was not available, but Eq. (17) should provide a reasonable representation for strains that do not exceed $10 \%$, which is the range relevant to conical and shallow spherical indentation. The elastic shear modulus of iridium is $217 \mathrm{GPa}$ and its Poisson's ratio has been taken to be 0.2 in the computations. When the material length parameters and the geometry of the indenter are specified, the finite element program can be used to compute the 


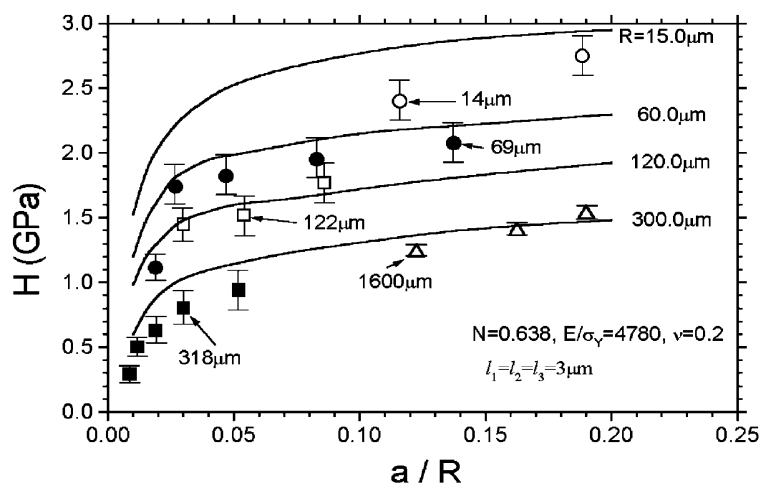

Fig. 10. Comparison of finite element results with the experimental results of Swadener et al. (2002) on spherical indentation of iridium. The numerical results were computed using the tensile stress-strain data (17) with $\ell_{1}=3 \mu \mathrm{m}$.

relation between $H$ and the indenter contact radius $a$. The computations have been carried out for the material specified by Eq. (17).

The computed results for spherical indentation are shown in Fig. 10 along with the experimental results of Swadener et al. (2002) for similar indenter radii. (Results for an indenter with a radius of $1600 \mu \mathrm{m}$ were not computed since they would be essentially indistinguishable from those for an indenter of radius $318 \mu \mathrm{m}$.) In the attempt to identify the applicable value of $\ell_{1}$, results were computed for several values of $\ell_{1}$ (with $\ell_{2}=\ell_{3}=\ell_{1}$ ). The curves computed with $\ell_{1}=3 \mu \mathrm{m}$ gave the reasonable fit to all the data seen in Fig. 10, and no further attempt was made iterate on $\ell_{1}$ to obtain a better fit. All aspects of the experimental trends for spherical indentation appear to be captured by the theory, although the spread between the theoretical results for $R=15$ and 60 is larger than that seen for the experimental data. ${ }^{1}$

The success of the theory is emphasized by the results in Fig. 11 for Berkovich indentation of iridium, computed for a conical indenter with $\beta=19.7^{\circ}$ and plotted as hardness versus indentation depth, $h=a \tan \beta$. In this case, the choice of $\ell_{1}=3 \mu \mathrm{m}$ was made from the outset in the numerical calculations. Agreement with the experimental data of Swadener et al. (2002) in Fig. 11 is remarkable. The agreement is probably fortuitous at indentation depths below $h=0.1 \mu \mathrm{m}$ since there is no evidence from applications to other problems that the theory is valid at a scale below a few tenths of a micron. (Note, however, that $h=0.1 \mu \mathrm{m}$ corresponds to an indentation diameter of $2 a=0.56 \mu \mathrm{m}$, which may be a more relevant measure of the size.) The data shown by large circles was taken with a micro-indenter while that represented by small circles was obtained with a nano-indenter. The smooth merge of the two sets of data speaks to the consistency of the two hardness test methods.

\footnotetext{
${ }^{1}$ The finite element mesh was refined several times to assure the accuracy of the results presented in Fig. 10. The results shown are for the finest mesh, and these differ only slightly from the results obtained from the next finest mesh.
} 


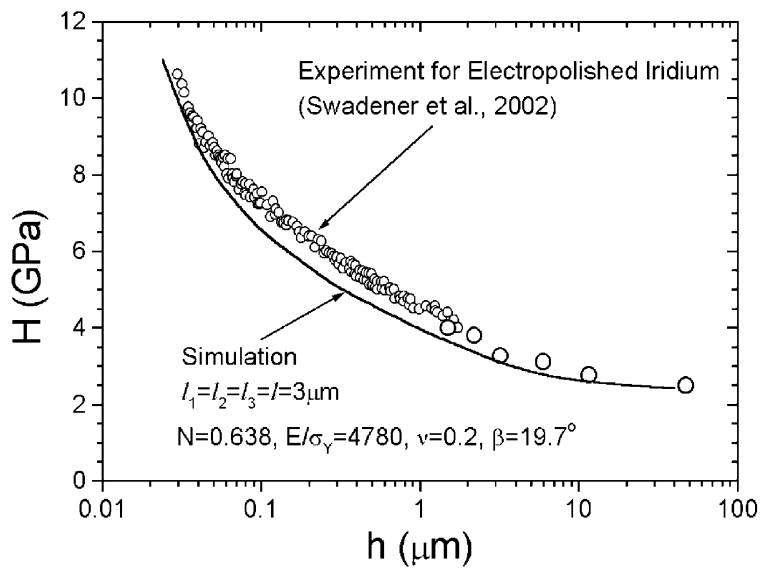

Fig. 11. Comparison of finite element results (computed using the tensile stress-strain data (17) with $\left.\ell_{1}=3 \mu \mathrm{m}\right)$ with the experimental results of Swadener et al. (2002) on conical indentation of iridium. Large circles are micro-indents; small circles are nano-indents.

\section{Concluding remarks}

The relation between indentation hardness and the stress-strain behavior is not simple, particularly for micron scale indents where size dependence becomes significant. Strain hardening, initial yield strain, indenter geometry, as well as indent size, are all important. Spherical indentation is more complicated to characterize than conical indentation. The spherical void approximation captures general trends reasonably well but inaccuracies emerge, particularly in the case of spherical indentation. Agreement between theoretical predictions based on strain gradient plasticity and indentation tests on iridium seen in Figs. 10 and 11 required detailed numerical computation. The spherical void approximation was not sufficiently accurate to bring out the excellent comparison between theory and experiment seen in these figures.

Crude approximations can be obtained by extending the simple connection discussed in Section 4.3, $H \cong 3 \sigma\left(\varepsilon_{\mathrm{r}}\right)$, between hardness and the uniaxial stress-strain curve proposed by Tabor (1951) to account for strain gradient effects. The idea is to replace the representative strain, $\varepsilon_{\mathrm{r}}$, by an effective strain measure, $E_{\mathrm{P}}$, in a form suggested by Eq. (1). The gradient of $\varepsilon_{\mathrm{r}}$ should scale as $c \varepsilon_{\mathrm{r}} / a$ such that new representative strain is $E_{\mathrm{p}}=\sqrt{\varepsilon_{\mathrm{r}}^{2}+\left(c \ell_{1} \varepsilon_{\mathrm{r}} / a\right)^{2}}$, where $c$ can be adjusted to fit data or numerical results. In this formula, $\varepsilon_{\mathrm{r}}$ is specified by the Tabor formulas depending on the whether a conical or spherical indenter is used. The trends that result from this formula are not unlike those obtained by Swadener et al. (2002) from their dislocation model. Certain of the trends are captured in an approximate manner, but the simple formula is incapable of accurate predictions of the theory such as those obtained by the full numerical calculations in Figs. 10 and 11.

Finally, both the spherical void approximation and the detailed finite element computations reveal that only one of the three material length parameters, $\ell_{1}$, is important in 
indentation. Moreover, indentation is probably the best method for determining this parameter experimentally. For iridium, tested by Swadener et al. (2002), $\ell_{1}=3 \mu \mathrm{m}$. Other metals, with higher initial yield strain and lower strain hardening, tend to have lower values of $\ell_{1}$, generally lying in the range from 0.25 to $1 \mu \mathrm{m}$ (Begley and Hutchinson, 1998).

\section{Acknowledgements}

The work of Y.W. was supported by the National Science Foundation of China through Grant 19925211 and by the Chinese Academy of Sciences through the "Bai Ren" project. The work of JWH was supported in part by Grant NSF DMR-0213805 and in part by the Division of Engineering and Applied Sciences, Harvard University.

\section{Appendix A}

\section{A.1. Constitutive aspects of the finite element method}

The variation of the functional defined in Eq. (5) with respect to $\left(\delta u_{i}, \delta \varepsilon_{\mathrm{P}}\right)$ can be put into the form

$$
\begin{aligned}
\delta \Phi= & \int_{V}\left\{\sigma_{i j} \delta \varepsilon_{i j}+\left[\frac{\sigma\left(\varepsilon_{\mathrm{P}}\right)}{E_{\mathrm{P}}}\left(\varepsilon_{\mathrm{P}}+\frac{1}{2} B_{i} \varepsilon_{\mathrm{P}, i}+C \varepsilon_{\mathrm{P}}\right)-\sigma_{\mathrm{e}}\right] \delta \varepsilon_{\mathrm{P}}\right. \\
& \left.+\frac{\sigma\left(\varepsilon_{\mathrm{P}}\right)}{E_{\mathrm{P}}}\left(A_{i j} \varepsilon_{\mathrm{P}, j}+\frac{1}{2} B_{i} \varepsilon_{\mathrm{P}}\right) \delta \varepsilon_{\mathrm{P}, i}\right\} \mathrm{d} V-\int_{S_{T}}\left(T^{0} \delta u_{i}+t^{0} \delta \varepsilon_{\mathrm{P}}\right) \mathrm{d} S,
\end{aligned}
$$

where the notation is defined in Section 2. The variables used in formulating the finite element scheme are $\left(u_{i}, \varepsilon_{\mathrm{P}}\right)$. Coefficients $B_{i}$ and $C$ depend on $m_{i j, k}$ which in turn depend on the gradient of the stress deviator. To obtain $s_{i j, k}$ in term of the strain gradient quantities, $\varepsilon_{i j, k}$ and $\varepsilon_{\mathrm{P}, k}$, write the deviator stress in term of the elastic strain deviator, $s_{i j}=2 G e_{i j}^{\mathrm{e}}$, with $G$ as the elastic shear modulus and $e_{i j}=\varepsilon_{i j}-\frac{1}{3} \varepsilon_{k k} \delta_{i j}$ as the deviator strain. From this relation, solve for the deviator stress as

$$
s_{i j}=H e_{i j},
$$

where $H=2 G /\left(1+3 G \varepsilon_{\mathrm{P}} / \sigma_{\mathrm{e}}\right)$. A direct calculation then gives

$$
s_{i j, k}=H e_{i j, k}-\frac{3 H^{2}}{2 \sigma_{\mathrm{e}}} e_{i j} \varepsilon_{\mathrm{P}, k}+\frac{3 H^{2} \varepsilon_{\mathrm{P}}}{2 \sigma_{\mathrm{e}}^{2}} e_{i j} \sigma_{\mathrm{e}, k}
$$

with

$$
\sigma_{\mathrm{e}, k}=\left[\frac{3 H s_{i j} e_{i j, k}}{2}-\frac{9 H^{2}}{4 \sigma_{\mathrm{e}}} s_{i j} e_{i j} \varepsilon_{\mathrm{P}, k}\right]\left[\sigma_{\mathrm{e}}-\frac{9 H^{2} \varepsilon_{\mathrm{P}}}{4 \sigma_{\mathrm{e}}^{2}} s_{i j} e_{i j}\right]^{-1} .
$$


The components above must be converted to the components for axisymmetric problems in cylindrical coordinates. The solution is obtained by iteration based on the variational equation, $\delta \Phi=0$. A finite element discretization of this equation is carried out. In outline, the iterative process is as follows. At any load, take as the first iteration either the conventional deformation theory solution (obtained numerically) or the converged solution at a neighboring load. Compute the stress gradient using Eq. (A.3) and the coefficients in the expression for $E_{\mathrm{P}}$. A linear iteration for increments in the nodal variables can be computed. Update the $\varepsilon_{i j}$, $\varepsilon_{\mathrm{P}}$ and their gradients, and repeat the iteration process until convergence is achieved. The number of iterative steps to achieve convergence (with relative change between successive steps falling below $10^{-4}$ ) is typically about 150 for $N=0.1$ and 120 for $N=0.2$.

\section{References}

Ashby, M.F., 1970. The deformation of plastically non-homogeneous alloys. Philos. Mag. 22, $399-424$.

Begley, M.R., Hutchinson, J.W., 1998. The mechanics of size-dependent indentation. J. Mech. Phys. Solids 46, 2049-2068.

Elmustafa, A.A., Stone, D.S., 2003. Nanoindentation and the indentation size effect: kinetics of deformation and strain hardening. J. Mech. Phys. Solids 51, 357-381.

Fleck, N.A., Hutchinson, J.W., 1997. Strain gradient plasticity, In: Hutchinson, J.W., Wu, T.Y. (Eds.), Advances in Applied Mechanics, Vol. 33. Academic Press, New York, pp. 295-361.

Fleck, N.A., Hutchinson, J.W., 2001. A reformulation of strain gradient plasticity. J. Mech. Phys. Solids 49, 2245-2271.

Fleck, N.A., Muller, G.M., Ashby, M.F., Hutchinson, J.W., 1994. Strain gradient plasticity: theory and experiment. Acta Metall. Mater. 42, 475-487.

Freund, L.B., Suresh, S., 2003. Thin Films and Layered Materials: Stress Deformation and Failure. Cambridge University Press, Cambridge, in press.

Gao, H., Huang, Y., Nix, W.D., Hutchinson, J.W., 1999. Mechanism based strain gradient plasticity: I-theory. J. Mech. Phys. Solids 47, 1239-1263.

Huang, Y., Xue, Z., Gao, H., Nix, W.D., Xia, Z.C., 2000. A study of micro-indentation hardness tests by mechanism-based gradient plasticity. J. Mater. Res. 15, 1786-1796.

Hutchinson, J.W., 2000. Plasticity at the micron scale. Int. J. Solids Struct. 37, 2225-2238.

Johnson, K.L., 1970. The correlation of indentation experiments. J. Mech. Phys. Solids 18, 115-126.

Ma, Q., Clarke, D.R., 1995. Size dependent hardness of silver single crystals. J. Mater. Res. 10, 853-863.

Marsh, D.M., 1964. Plastic flow in glass. Proc. R. Soc. A 279, 420.

Nix, W.D., Gao, H., 1998. Indentation size effects in crystalline materials: a law for strain gradient plasticity. J. Mech. Phys. Solids 46, 411-425.

Stelmashenko, N.A., Walls, M.G., Brown, L.M., Milman, Y.V., 1993. Microindentations on W and Mo oriented single crystals: an STM study. Acta Metal. Mater. 41, 2855-2865.

Stolken, J.S., Evans, A.G., 1998. A microbend test method for measuring the plasticity length scale. Acta Mater. 46, 5109-5115.

Swadener, J.G., George, E.P., Pharr, G.M., 2002. The correlation of the indentation size effect measured with indenters of various shapes. J. Mech. Phys. Solids 50, 681-694.

Tabor, D., 1951. Hardness of Metals. Clarendon Press, Oxford.

Timoshenko, S., Goodier, J.N., 1970. Theory of Elasticity, 3rd Edition. McGraw-Hill, New York.

Xue, Z., Huang, Y., Hwang, K.C., Li, M., 2002. The influence of indenter tip radius on micro-indentation hardness. J. Eng. Mater. Technol. 124, 371-379. 